\title{
Untersuchung der kosmischen Strahlenschauer mit Hilfe einer großen Wilson-Kammer*
}

\author{
Von Martin Deutschmann \\ Aus dem Physikalischen Institut der Universität Freiburg i. Br. \\ (Z. Naturforschg. 2a, 61-69 [1947]; eingegangen am 18. Dezember 1946)
}

\begin{abstract}
Mit einer fast 100 Liter fassenden Wils on-Kammer wurden 200 ausgedehnte Schauer aufgenommen. Es wurde die Häufigkeitsverteilung der Strahlendichten ermittelt. Sie stimmt mit entsprechenden Messungen $\mathrm{H}$ of f man $\mathrm{n}$ scher Stöße überein und zeigt, daß letztere sich überwiegend aus schwach ionisierenden Teilchen zusammensetzen müssen. Schwere Teilchen waren nur in sehr geringer Zahl feststellbar. Ferner wurden die Einfallsrichtungen der Schauer gemessen. Die Richtungsverteilung unterscheidet sich charakteristisch von derjenigen der Einzelstrahlen; sie läßt Schlüsse auf die Absorbierbarkeit und Natur der schauererzeugenden Primärstrahlung zu. Die Schauerteilchen lösten im Kammergas zahlreiche Sekundärelektronen aus. Das Energiespektrum dieser Elektronen verläuft steiler, als nach der Stoßtheorie zu erwarten ist.
\end{abstract}

$\mathrm{S}^{\mathrm{e}}$ eit den Experimenten von $\mathrm{K}$ ol hörster ${ }^{1}$ und A u ger ${ }^{2,3}$ weiß man von der Existenz ausgedehnter kosmischer Strahlenschauer, die sich durch enorm hohe Teilchenenergien auszeichnen. Da unsere derzeitige Kenntnis dieser Schauer überwiegend aus Zählrohrmessungen gewonnen ist, erscheinen Untersuchungen mit anderen Methoden, z. B. mit Hilfe der Wils on Kammer, sehr wünschenswert. Über derartige Wilson-Aufnahmen ist erst wenig veröffentlicht worden ${ }^{3,4,5}$. Auch wurden bisher nur relativ kleine Kammern verwendet. Die vorliegende Arbeit bringt daher Aufnahmen mit einer besonders geeigneten, sehr großen Kammer von nahezu 100 Liter Inhalt. Es zeigt sich, daß man an Hand solcher Aufnahmen in der Lage ist, quantitative Aussagen über zahlreiche, den Schauer betreffende Fragen zu machen.

* Gekürzte Dissertation T.H. Berlin 1944.

1 W. Kolhörster, I. Mat thes u. E. Web e r, Naturwiss. 26, 576 [1938].

${ }^{2}$ P. A u ger, R. M a ze u. T. Grivet-Meyer, C. R. hebd. Séances Acad. Sci. 206, 1721 [1938]; P. A u ger u. R. M a ze, C. R. hebd. Séances Acad. Sci. 207, 228 [1938].

${ }^{3} \mathrm{P}$. A u ger, R. Maze, $\mathrm{P}$. Ehrenfest u. A. Freon, J. Physique Radium 10, 39 [1939].

4. Ján os s y u. B. C. L o vell, Nature [London] 142, 716 [1938].

\section{Die Wilson-Kammer}

Die nach Plänen von Hrn. Prof. Dr. H. G e i g e r erbaute Nebelkammer ${ }^{6}$ ist in Abb. 1 skizziert. Sie besteht aus einem zylindrischen Kammerraum von

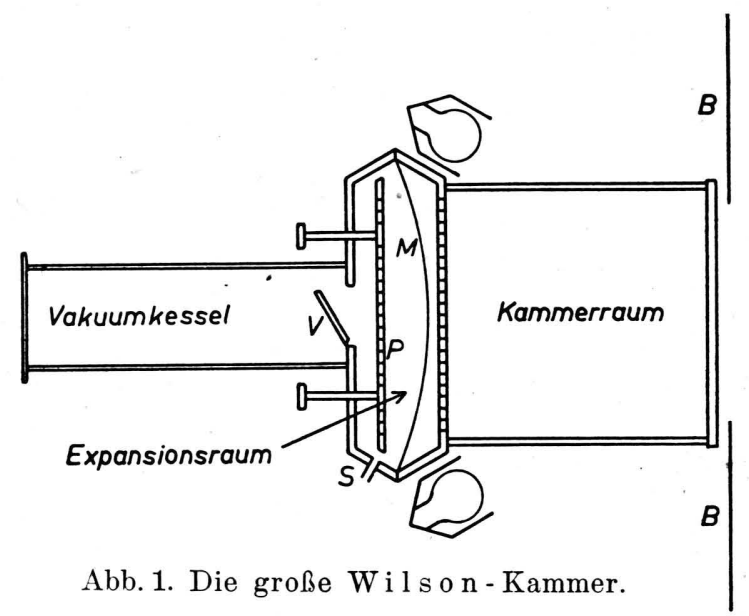

$50 \mathrm{~cm}$ Durchmesser und $50 \mathrm{~cm}$ Tiefe, der durch einen Glaszylinder und eine Glasplatte gebildet wird. Die Kammer ist liegend aufgebaut und wird von der Seite her betrachtet. Expandiert wird mit Hilfe einer

${ }^{5}$ B. C. L o vell u. J. G. Wil s o n, Nature [London] 144, 863 [1939].

${ }^{6}$ M. De utschmann, unveröff. Diplomarbeit T.H. Berlin [1941]. 
Gummimembran $M$, die zwischen zwei große Aluminiumteller eingespannt ist. Der vordere Teller ist auf seiner ganzen Fläche gelocht und wird im Kammerraum von einem durchlässigen schwarzen Trikotstoff bedeckt. Den Expansionshub begrenzt die verstellbare, ebenfalls gelochte Anschlagplatte $P$. Hinter ihr befindet sich das Ventil $V$, welches elektrisch betätigt wird. Als Kammerfüllung hat sich alkoholgesättigte Luft von Atmosphärendruck bewährt. Beleuchtet wird mit zehn 500-Watt-Glühlampen, die bei photographischer Aufnahme mit 1,5-facher Nennspannung brennen. 1,3 $\mathrm{m}$ vor der Kammer stehen, durch den Blendschutz $B$ vor direktem Lampenlicht geschützt, zwei Leica-Apparate und nehmen die Nebelspuren stereoskopisch auf. Die Auslösung der Kameras erfolgt erst 0,2 bzw. 0,35 sec nach der Expansion (je nachdem, ob die Kammer zählrohrgesteuert ist oder nicht). Bis zu diesem Zeitpunkt haben sich die

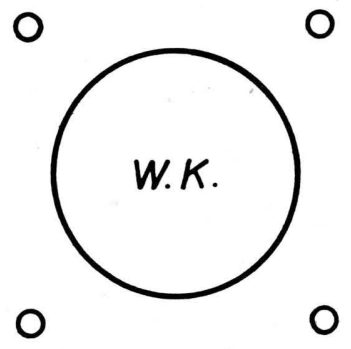

Abb. 2. Die Zählrohranordnung.

Nebēltröpfchen zu ihrer günstigsten Größe entwickelt. Die Lampen erreichen schon nach 0,1 sec volle Lichtstärke.

Die Abmessungen der Kammer sind sehr groß gewählt worden, um für die Aufnahme von Ultrastrahlen günstige Bedingungen zu schaffen. So ist es z. B. möglich, ohne Zählrohrsteuerung zu arbeiten, solange nicht besonders seltene Prozesse interessieren. Von den gefürchteten Expansionswirbeln ist die Kammer trotz ihres großen Volumens völlig frei. Eine gute Konstanz der Raumtemperatur ist jedoch erforderlich. Aus diesem Grund war es nötig, die Kammer im gleichmäßig temperierten Erdgeschoß des Institutes aufzustellen.

Die großen Schauer, die nur 2- bis 3-mal in der Stunde einfielen, erfaßte eine Zählrohranordnung nach Abb. 2. Vier mit $60 \mathrm{~cm}$ Abstand angebrachte Rohre von $1 \mathrm{~m}$ Länge und $3 \mathrm{~cm}$ Durchmesser mußten gleichzeitig vom Schauer getroffen werden. Die Koinzidenz wurde vom Verstärker einem kräftigen Thyratron zugeleitet, das nach der von B la cket t ${ }^{7}$ angegebenen Methode das Expansionsventil, die Beleuchtung und die Photoapparate steuerte.

Die insgesamt 200 Aufnahmen wurden folgendermaßen ausgewertet: Um die Lage der Nebelspuren räumlich zu vermessen, wurden auf die Filme unter dem Stereoskop Aufnahmen eines Raumgitters aufgelegt und die mit der Nebelspur sich deckenden

7 P. M. S. B l a c k ett, Proc. Roy. Soc. [London] Ser. A 146, 281 [1934].
Gitterkoordinaten abgelesen. Das aus dünnen Fäden hergestellte Gitter war zuvor in die Kammer eingeschoben und bei unveränderter Kamerastellung aufgenommen worden, so daß sich die Testfilme genau mit den Aufnahmen deckten.

\section{Die Ursprungsorte der Schauer- strahlen}

Um festzustellen, ob es sich bei den Aufnahmen um Luft- oder Gebäudeschauer handelt, wurden die Filme zunächst hinsichtlich der Entstehungsorte der Schauerteilchen durchgemustert. Es ergab sich, daß 95\% aller Schauer nicht in der Decke oder in den Wänden des Versuchsraumes entstanden sein konnten, da die Teilchenbahnen keinen meßbaren Divergenzwinkel aufwiesen. Entweder liefen die Bahnen völlig parallel, oder die Teilchen kamen von mehreren, weit auseinander liegenden Entstehungsorten. Nur zehn Aufnahmen deuten auf einen möglichen Ursprung des Schauers bzw. des in der Kammer beobachtbaren Teils eines solchen in der Zimmerdecke hin. Wenn wir annehmen, daß in den beiden Decken des Ober- und des Dachgeschosses etwa gleich viel Schauer entstanden sind wie in der Zimmerdecke, so muß immer noch die überwiegende Mehrzahl der Schauer (sicher mehr als $80 \%$ ) nicht im Gebäude, sondern in der Luft darüber entstanden sein.

Allerdings hat sich die Partikelzahl der Schauer im Gebäude stark vermehrt. Dies beweist folgender Versuch: Die zur Steuerung der Kammer verwendete Zählrohranordnung ergab unter freiem Himmel eine 4,5-mal kleinere Koinzidenzzahl als im Versuchsraum. Dieses Ergebnis ist erklärlich, wenn man bedenkt, daß mit abnehmender Teilchendichte die Treffwahrscheinlichkeit der Zählrohre stark sinkt. Der Effekt steht übrigens in gutem Einklang mit Messungen von Auger ${ }^{2,3}$.

\section{Die Strahlendichte im Schauer}

Wie die hier wiedergegebenen Aufnahmen (s. Tafel) zeigen, ist die Strahlendichte der Schauer individuell sehr verschieden. Während man durchschnittlich 16 Bahnen im Kammerraum beobachtet, enthält beispielsweise Abb. 4 rund 140 Teilchenspuren. Dieser Befund, der dadurch zustande kommt, daß Schauer an und für sich wechselnder Gesamtteilchenzahl die Kammer mit ihren Kern- oder Randbereichen durchsetzen, ist in Tab. 1 zusammengestellt. Man erkennt, daß eine geringe Teil- 
chendichte sehr häufig vorkommt, und daß nach großen Dichten hin eine stetige Abnahme der Häufigkeit erfolgt. Die bei sehr kleinen Teilchendichten $(N<5)$ auftretende Ausnahme ist nur scheinbar; sie ist durch die Meßtechnik bedingt, wie weiter unten noch beschrieben wird.

\begin{tabular}{|c|c||c|c|}
\hline $\begin{array}{c}N \\
\text { Zahl der } \\
\text { Teilchen }\end{array}$ & $\begin{array}{c}Z \\
\text { Zahl der } \\
\text { Aufnahmen }\end{array}$ & $\begin{array}{c}N \\
\text { Zahl der } \\
\text { Teilchen }\end{array}$ & $\begin{array}{c}Z \\
\text { Zahl der } \\
\text { Aufnahmen }\end{array}$ \\
\hline $0-5$ & 28 & $41-50$ & 1 \\
$6-10$ & 65 & $51-60$ & 6 \\
$11-15$ & 43 & $61-70$ & 2 \\
$16-20$ & 25 & $71-80$ & 1 \\
$21-30$ & 16 & $81-90$ & 1 \\
$31-40$ & 10 & $141-150$ & 1 \\
\hline
\end{tabular}

Tab. 1. Die Teilchendichte der Aufnahmen.

Die Daten der Tab. 1 legen einen Vergleich mit Messungen $\mathrm{H}$ offmannscher .Stöße in ungepanzerten Ionisationskammern ${ }^{8-12}$ nahe, denn sie entsprechen der Abhängigkeit Stoßhäufigkeit Stoßgröße. Bevor wir jedoch quantitativ vergleichen, müssen wir einen prinzipiellen Unterschied beider Meßmethoden berücksichtigen: Jede zählrohrgesteuerte Apparatur spricht im Gegensatz zur Ionisationskammer im Fall geringer räumlicher Strahlendichte nur mit begrenzter Wahrscheinlichkeit an. Es werden also unter unseren Wilson-Aufnahmen die teilchenarmen in zu geringer $\mathrm{Zahl}$ vertreten sein. Glücklicherweise läßt sich dieser Effekt rechnerisch erfassen: Bekanntlich beträgt die Treffwahrscheinlichkeit $w_{1}$ eines Schauers der Teilchendichte $\varrho\left[\mathrm{cm}^{2}\right]$ auf ein Zählrohr der Fläche $f: w_{1}=1-e^{-\varrho f}$. Die Wahrscheinlichkeit einer Vierfachkoinzidenz lautet dann $w_{4}=\left(1-e^{-\varrho f}\right)^{4}$, wenn man die Strahlendichte im Bereich der vier Rohre (alle mit gleichem $f$ ) als konstant ansetzt. Das $w_{4}$ ist ohne weiteres zu errechnen, da die Zählrohrfläche $f=300 \mathrm{~cm}^{2}$ bekannt ist und $\varrho$ die Teilchenzahl $N$, bezogen auf den Kammerquerschnitt von $2000 \mathrm{~cm}^{2}$, bedeutet ${ }^{13}$.

Abb. 5 bringt nun die auf Treffwahrscheinlichkeit Eins korrigierten Schauerhäufigkeiten der Tab.1. Es ist die integrale Darstellung gewählt,

8 H. S ch m id, Z. Physik 117, 452 [1941].

9 H. C a rmichael u. C.N. Chou, Nature [London] 144, 325 [1939].

${ }_{10}$ C. G. Montgomery u. D. D. Montgomery, Rev. mod. Physics 11, 255 [1939]. d.h. es ist die Zahl der Aufnahmen angegeben, deren Teilchenmenge einen Mindestwert $N$ übertrifft. Als Fehlergrenzen sind die mittleren statistischen Fehler $(\sqrt{H})$ eingezeichnet. Zum Vergleich enthält Abb.5 auch die unkorrigierten Häufigkeiten $(+)$. Man sieht, daß die Korrektur nur unterhalb von $N=20$ Teilchen von Bedeutung ist. Nach der doppeltlogarithmischen Darstellung nimmt die Schauerzahl mit wachsender Strahlendichte nach einem Potenzgesetz ab:

$$
H=\text { const } \cdot N^{-s} \text {. }
$$

Der Exponent ergibt sich aus den Meßpunkten zu $s=1,95 \pm 0,1$. Dieses Ergebnis können wir nun mit den Stoßmessungen vergleichen.

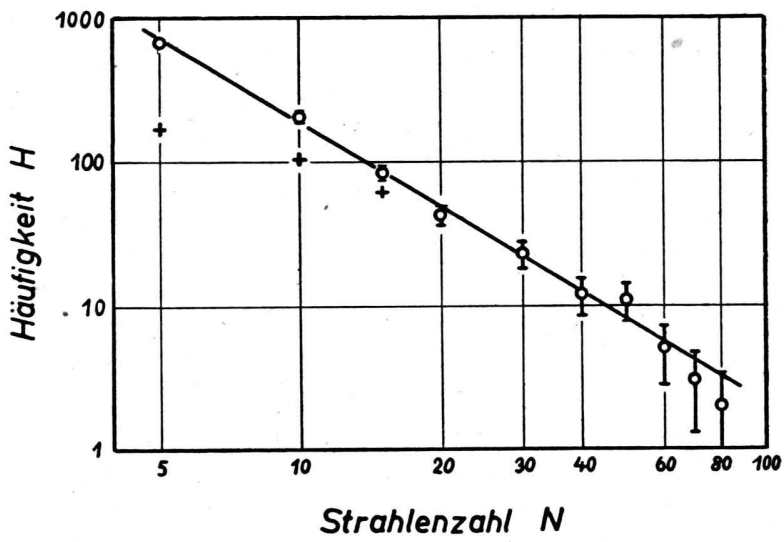

Abb. 5. Zahl der Aufnahmen mit mehr als $N$ Teilchen.

Die Stoßmessungen bestätigen das Potenzgesetz (1), wenn man die Stoßgröße der Partikelzahl $N$ proportional setzt. Für den Exponenten $s$ sind bisher nicht ganz einheitliche Werte gemessen worden ${ }^{8-12}$. Sie schwanken für unbedeckte Kammern zwischen $s=2,1$ und $s=3,3$. Mit wachsender Materieschicht über der Kammer fällt der Exponent allmählich ab, wie es z. B. aus den Messungen von $\mathrm{H}$. Schmid ${ }^{8}$ deutlich hervorgeht. Dieses Abfallen erklärt zwanglos den etwas niedrigen Wert $s=1,95$ unserer Messung als Einfluß der Gebäudedecken. Die Schmidschen Kurven lassen obendrein erkennen, daß der Abfall von $s$ als ein Anwachsen der Zahl teilchenreicher Schauer zu verstehen ist. Dies bestätigt

11 M. Schein u. P. S. Gill, Rev. mod. Physics 11, 267 [1939].

12 R. E. L a p p, Physic. Rev. 64, 129 [1943].

13 Der Kammerquerschnitt beträgt eigentlich $2500 \mathrm{~cm}^{2}$, er ist aber wegen des Blendschutzes nicht vollständig sichtbar. 
das Ergebnis des vorigen Paragraphen, daß sich nämlich die Teilchenzahl der Schauer im Gebäude vermehrt hat, daß neue Schauer aber kaum erzeugt wurden.

Es kann also ein befriedigendes Übereinstimmen der Wils on-Kammer- und der Ionisationskammermessungen festgestellt werden. Dabei ist $\mathrm{zu}$ bemerken, daß vorliegende Untersuchung gerade auch den Bereich geringer Teilchendichten erfaßt, der für Ionisationskammern aus meßtechnischen Gründen - wegen des statistischen Schwankens der Galvanometerausschläge - nicht so leicht zugänglich ist. Die Übereinstimmung der Exponenten $s$ spricht dafür, daß die H offmannschen Stöße in nicht gepanzerten Kammern durch die gleichen Schauer hervorgerufen werden, wie sie hier in der Nebelkammer beobachtet wurden, d. h. durch Schauer zahlreicher, schwach ionisierender Teilchen. Wohl können diese Schauer mitunter schwere Teilchen enthalten ( siehe nächster Abschnitt), doch erscheint deren Zahl zu gering, um sich in der Stoßverteilungskurve merklich auszuwirken.

\section{Stark ionisierende Teilchen i m Sch a u er}

Auf fast allen Aufnahmen sind kurze, meist diffuse Bahnen sehr stark ionisierender Teilchen zu sehen. Diese Teilchen gehören nicht den Schauern an, es sind normale $\alpha$-Strahlen der stets vorhandenen radioaktiven Beimengungen der Luft. Andererseits zeigen 9 Aufnahmen scharfe Spuren stärker ionisierender Strahlen von wesentlich größerer Reichweite (s. Tafel, Abb.6). Es dürfte sich dabei um energiearme Protonen handeln. Daß diese Strahlen mit den Schauern gekoppelt sind, folgt aus zweierlei Beobachtungen: Einmal müssen sie wegen der Schärfe ihrer Bahnspur den Schauern zeitlich zugeordnet werden. Zweitens spricht ihre Häufigkeit dafür, daß sie nicht zufällig sind; denn unter 400 Aufnahmen von Einzelstrahlen mit ungesteuerter Kammer befand sich nur ein einziges stärker ionisierendes Teilchen. Es ist bemerkenswert, daß die schweren Teilchen ganz unabhängig von der Strahlendichte der Schauer erschienen. Auch war keine Einfallsrichtung merklich bevorzugt. Letzteres stimmt mit Beobachtungen von Anders on und Ned-

${ }_{14}$ C. D. A nderson u. S. H. Nedderm e y e r, Physic. Rev. 50, 263 [1936].

15 E. B a g g e, Ann. Physik 39, 535 [1941]. d e r m e y e $\mathrm{r}^{14}$ an kleinen Schauern überein. Es ist auch nach den von $\mathrm{Bag} g \mathrm{e}^{15}$ diskutierten Kernprozessen verständlich.

Die Einfallsrichtung ausgedehnter $\mathrm{Sch}$ auer

Die strahlenreicheren Schauer der vorliegenden Aufnahmeserie zeigen überwiegend parallel verlaufende Teilchenbahnen, so daß man von einer definierten Einfallsrichtung der Schauer sprechen kann. Die stereoskopischen Aufnahmen erlaubten, die Einfallsrichtungen räumlich festzulegen, d.h. die wahren Winkel gegen die Vertikale zu messen. Die Tab. 2 gibt in Spalte 2 an,

\begin{tabular}{|c|c|c|c|c|}
\hline \multirow{2}{*}{$\begin{array}{c}1 \\
\text { Winkel } \vartheta \\
\text { gegen die } \\
\text { Vertikale }\end{array}$} & & \multicolumn{2}{|c|}{ Zahl der Schauer } & \\
\hline & $\begin{array}{c}\text { be- } \\
\text { obachtet }\end{array}$ & $\begin{array}{l}\text { kor- } \\
\text { rigiert }\end{array}$ & $\begin{array}{l}\text { pro Raum- } \\
\text { winkel- } \\
\text { einheit }\end{array}$ & $\begin{array}{l}\text { stärke } \\
\text { in } \mathrm{m}\end{array}$ \\
\hline $\begin{array}{r}0-10 \\
10-20 \\
20-30 \\
30-40 \\
40-50 \\
50-60 \\
60-70 \\
70-80 \\
80-90\end{array}$ & $\begin{array}{r}38 \\
21 \\
17 \\
8 \\
3 \\
4 \\
5 \\
5 \\
\mathbf{2}\end{array}$ & $\begin{array}{c}30,5 \\
21,5 \\
18,5 \\
9,5 \\
4 \\
6,5 \\
10 \\
11 \\
6,5\end{array}$ & $\begin{array}{c}349 \\
83 \\
43,7 \\
16,5 \\
5,7 \\
7,9 \\
11 \\
11,4 \\
6,5\end{array}$ & $\begin{array}{c}0,4 \\
1,3 \\
1,7 \\
1,9 \\
2,2 \\
2,4 \\
2,6 \\
2,9 \\
?\end{array}$ \\
\hline
\end{tabular}

Tab. 2. Die Richtungsverteilung der Schauer.

wieviel Schauer beobachtet wurden, deren Einfallsrichtung einen Winkel \& gegen die Vertikale bildete. Da Schauer aller Himmelsrichtungen gezählt sind, beziehen sich die Häufigkeiten nicht auf gleiche, sondern mit $\sin \vartheta$ anwachsende Raumwinkelzonen. Bevor wir auf gleiche Raumwinkel umrechnen, soll an den Daten von Spalte 2 noch eine Korrektur angebracht werden, die sich wegen der nicht vollständigen Kugelsymmetrie der Zählrohranordnung als notwendig erwiesen hat. Es muß berücksichtigt werden, daß vertikal einfallende Schauer etwas bevorzugt waren, da sie unter Umständen bereits mit zwei Strahlen alle vier Rohre treffen konnten. Unter großem Zenitwinkel ankommende Schauer waren hingegen benachteiligt, denn sie fanden je nach Himmelsrichtung durch Projektion verkleinerte Zählrohrflächen vor. Den Richtungseinfluß der hier verwendeten Zählrohranordnung hat Wi t t ${ }^{16}$ experi-

${ }^{16} \mathrm{H}$. R. W it t, unveröff. Diplomarbeit T.H. Ber$\operatorname{lin}[1944]$. 
Ah). 3. Ein schauer von der am häufigsten beobachteten Art. Relativ wenige Partikeln (etwa 20) fallen fast senkrecht ein. Die Bahnen deuten nicht alle auf einen gemeinsamen Crsprungsort hin. Aulier den spuren des schauer. sieht man zahlreiche Bahnen energiearmer Elektronen. Sie sind z. Tl. durch Diffusion verbreitert, d.h. ıl : Teilchen haben die Kammer zu va...hiedenen Zeiten unabhängig vom Scharer durchquert.

Abb. 4. Ein hesonders strahlenreicher Schauer mit etwa 140 Teilchen im Kammerraum. Fire durch das Kammerfeld aufgespa!1 ne Spur eines $\alpha$-Strahls gehört nicht dem schauer an.

Ab.6. Ein stark ionisierendes Teilchen, vermutlich ein Proton, durchquert die Mitte des Kammerraumes.

Abb. 7. Ein fast waagerecht einfallender Schauer.

Abb. 10. In der durch Pfeile markierten Stelle wird ron einem Schauerstrahl ein Sekundärelektron ausgelöst.
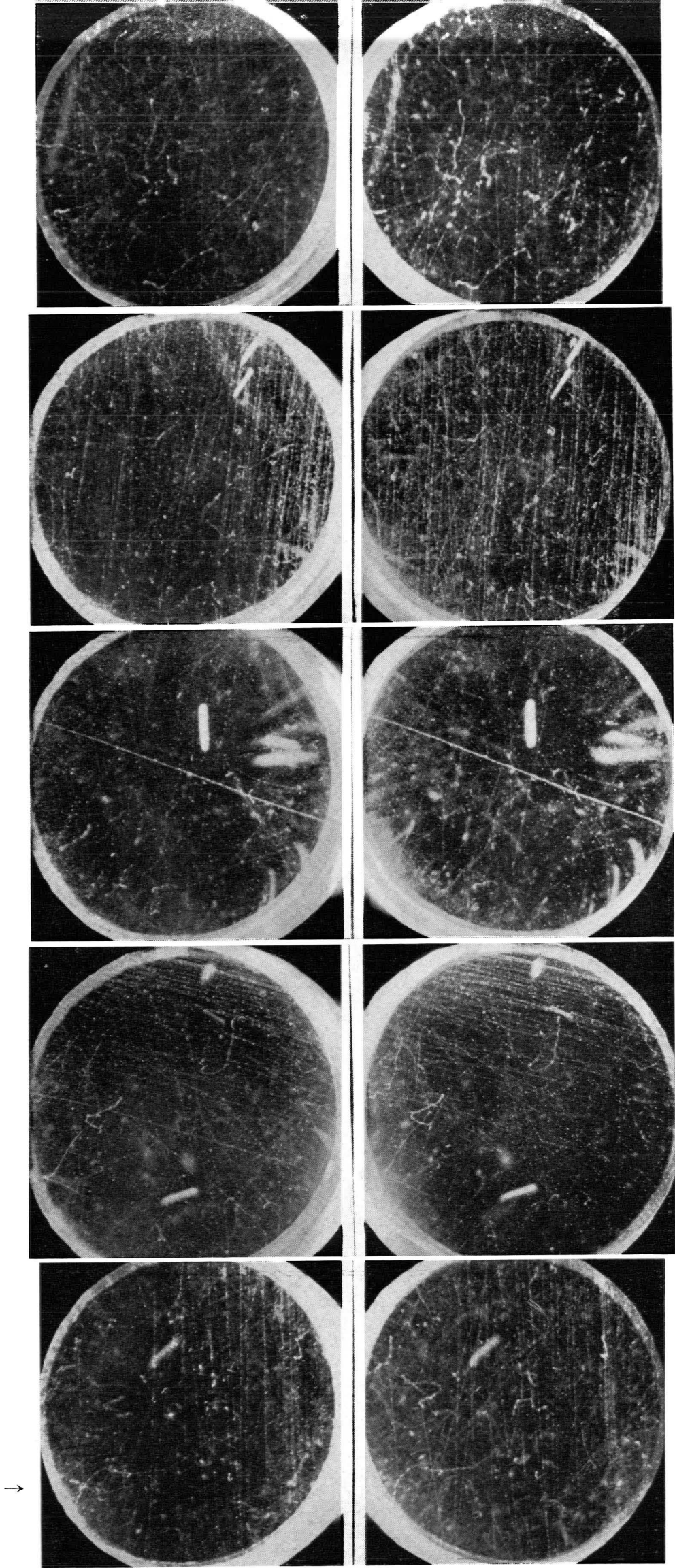
noch wenig abnimmt. Es liegen zwar für diesen Ast noch keine weiteren direkten Messungen vor, doch kann man als qualitative Bestätigung Ionisationskammeruntersuchungen von $\mathrm{Carmichael}$ und $\mathrm{Chou}^{9}$ bewerten. Nach den Kurven dieser Autoren sinkt die Stoßzahl unter einer Schicht von $30 \mathrm{~m}$ Erdboden, je nach Stoßgröße, um eine bis zwei Zehnerpotenzen ab. Ein Faktor gleicher Größenordnung läßt sich aus Abb. 8 errechnen. Wäre jedoch in Abb. 8 der steile Ast bis zu großen Tiefen gültig, bzw. existierte der flache Zweig nicht, so hätten $\mathrm{C}$ a r m i ch a e l und $\mathrm{Ch}$ o u $30 \mathrm{~m}$ unter der Erde nur den Bruchteil von $10^{-14}$ der tatsächlich gefundenen Stöße, also keinen einzigen mehr, registrieren dürfen.

Aus der guten Übereinstimmung mit anderen Messungen darf man schließen, daß es gerechtfertigt ist, die beobachtete Richtungsverteilung als Schichtdickenabhängigkeit zu deuten. Die in der Kammer gemessene Bahnrichtung der Schauerteilchen stimmt demnach mit der Einfallsrichtung des primären Teilchens überein und gibt zudem noch dessen Weg durch die Atmosphäre an. Daraus folgt weiter, daß die Winkelstreuung der Teilchen im ausgedehnten Schauer gering sein muß - übereinstimmend mit der Theorie ${ }^{\mathbf{2 0}, \mathbf{2 1}}$.

Es liegt die Vermutung nahe, daß sich in den beiden so deutlich voneinander abgegrenzten Kurvęnästen der Abb. 8 zweierlei Primärstrahlungen ausgedehnter Schauer äußern. Der steile Ast verläuft angenähert so, wie er für weiche Primärteilchen zu erwarten ist. Sein Abfall sollte allerdings nach der Kaskadentheorie ${ }^{22,23}$ eher etwas steiler ${ }^{24}$ erfolgen, wenn man annimmt, daß alle Primärteilchen aus einer einheitlichen Schicht, z. B. von der Atmosphärengrenze, kommen. Der Kurventeil bei dicken Schichten hingegen kann seine Entstehung nur einer durchdringenden Primärstrahlung verdanken. Er fällt wohl etwas flacher ab, als es die Absorptionsgesetze $^{22}$ der Mesonen fordern. Die Meßpunkte scheinen sogar auf ein breites Maximum (analog dem zweiten Maximum der Rossi-Kurve?) hinzuweisen. Irgendwelche Schlüsse sind jedoch wegen der begrenzten Meßgenauigkeit nicht mög-

20 H. E u l e r u. H. Wergeland, Naturwiss. 27. 484 [1939].

21 G. $\mathrm{Mol}$ i ère in W. He is e n berg, ,Vorträge über kosmische Strahlung“, Springer, Berlin [1943].

${ }^{22}$ H. Eule r u. W. H e i s e n be rg, Ergebn. exakt. Naturwiss. 17, 1 [1938]. lich. Die genauere Untersuchung des flachen Kurvenastes soll einer späteren Arbeit vorbehalten bleiben.

Aus Abb. 8 lassen sich noch einige Daten ablesen. Z. B. kann man aus der Schichtdickenabhängigkeit rückwärts wieder die Richtungsverteilung der Schauer $\left[F\left(z_{0}, \vartheta\right)\right.$ nach Fußnote $\left.{ }^{19}\right]$ errechnen, nun aber für beliebige Höhen $z_{0}$. In Abb. 9 ist dies für Meereshöhe $\left(z_{0}=1030 \mathrm{~g} / \mathrm{cm}^{2}\right)$ geschehen. Die Kurve entspricht der ursprünglich gemessenen Verteilung, nur ist jetzt der Einfluß der Gebäudewände rechnerisch beseitigt. Die

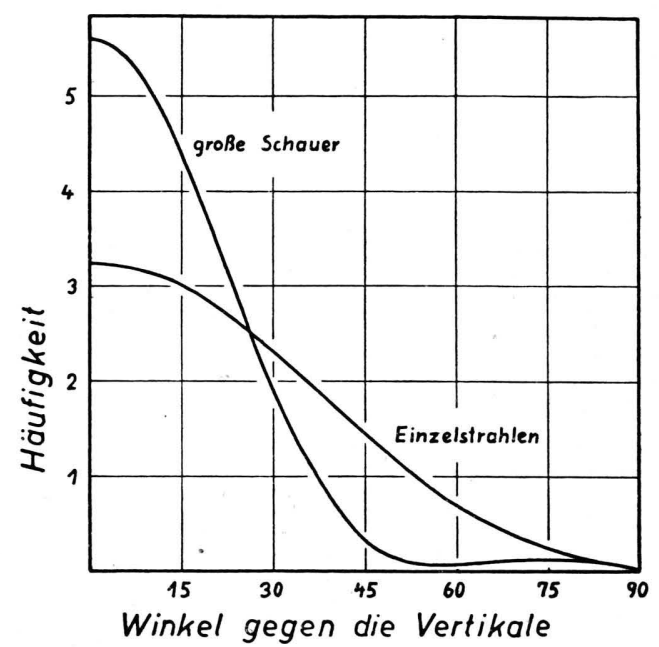

Abb. 9. Die Richtungsverteilung ausgedehnter Schauer in Meereshöhe.

Kurve unterscheidet sich sehr deutlich von der Winkelverteilung der Einzelstrahlen, welche bekanntlich einer $\cos ^{2} \vartheta$-Funktion ähnelt. Weiterhin kann Abb. 8 entnommen werden, zu welchem Prozentsatz in verschiedenen Höhenlagen durchdringende Teilchen große Schauer hervorrufen. Man muß dann nur für die gewünschte Höhe den Anteil beider Kurvenäste gesondert berechnen. In Meereshöhe erhält man beispielsweise $12 \%$ durchdringende Primärteilchen. Mit wachsenden Höhen nimmt dieser Anteil rasch ab, wogegen hinter dicken Schichten große Schauer nur noch von durchdringenden Teilchen erzeugt werden.

23 W. H e is e n b e r g, ,Vorträge über kosmische Strahlung“, Springer, Berlin [1943].

${ }^{24} \mathrm{H}$ i l b e r r y ${ }^{\mathbf{1 8}}$ findet durch Rechnung ebenfalls einen steileren (nicht-exponentiellen) Abfall und deutet die Abweichung des Experiments in tieferen Lagen als Folge durchdringender Teilchen. Quantitativ ist diese Deutung mit unserer Messung nicht verträglich. 
Sekundärelektronen der Schauer. teilehen

Beim näheren Betrachten der vorliegenden Aufnahmen (z. B. Abb. 10 der Tafel) erkennt man, daß von den Schauerstrahlen im Gas des Kammerraumes häufig kleine Sekundärbahnen, sogenannte $\delta$ Strahlen, ausgehen. Die große Zahl der aufgenommenen Bahnspuren ermöglichte es, die Sekundärteilchen eingehender $\mathrm{zu}$ untersuchen. Es wurden auf insgesamt $31100 \mathrm{~cm}$ Bahnlänge 174 solcher Sekundärelektronen beobachtet, deren Reichweite ${ }^{25} 6 \mathrm{~mm}$ und mehr betrug. Tab. 3 bringt

\begin{tabular}{|c|c|c|c|}
\hline $\begin{array}{c}\text { I. Stoßende Teilchen } \\
\text { Schauerstrahlen } \\
\text { Länge der Primärbahn } \\
31100 \mathrm{~cm}\end{array}$ & \multicolumn{2}{|c|}{$\begin{array}{c}\text { II. Stoßende Teilchen } \\
\text { Einzelstrahlen } \\
\text { Länge der Primärbahn } \\
7300 \mathrm{~cm}\end{array}$} \\
\hline $\begin{array}{c}\text { Reichw. R } \\
\text { in [cm] }\end{array}$ & $\begin{array}{c}\text { Sek.-El.- } \\
\text { Zahl }\end{array}$ & $\begin{array}{c}\text { Reichw. R } \\
\text { in [cm] }\end{array}$ & $\begin{array}{c}\text { Sek.-El.- } \\
\text { Zahl }\end{array}$ \\
\hline $0,6-1$ & 65 & $0,5-0,8$ & 5 \\
1 - 1,5 & 36 & $0,8-1,3$ & 4 \\
$1,5-2$ & 17 & $1,3-1,7$ & 2 \\
$2-3$ & 19 & $1,7-2,6$ & 4 \\
$3-4$ & 11 & 2,6 & 3 \\
$4-5$ & 7 & & \\
$5-6$ & 4 & & \\
$6-8$ & 5 & & \\
$8-11$ & 4 & & \\
$11-16$ & 2 & & \\
16 & 4 & & \\
\hline
\end{tabular}

Tab. 3. Sekundärelektronen, im Gas der Kammer ausgelöst.

im einzelnen die Zahl der Sekundärteilchen in Abhängigkeit von deren Reichweite. Die Tabelle enthält auch Daten über Sekundärelektronen von Einzelultrastrahlen, die der auf S. 64 erwähnten Aufnahmeserie bei ungesteuerter Kammer entnommen sind. Bei Einzelstrahlen ist die Zahl der $\delta$-Bahnen geringer. Auf $8400 \mathrm{~cm}$ Primärbahn wurden nur 18 Sekundärteilchen von $6 \mathrm{~mm}$ gemessener Mindestreichweite gezählt. Die Einzelstrahlen hatten übrigens die Kammer unter etwas vermindertem Druck passiert; sie sind im Gegensatz zu den Schauern erst während der eigentlichen Bereitschaftszeit eingefallen, die im Moment der Expansion beginnt und bei dieser großen Kammer ca. ${ }^{1 / 3}$ sec dauert. Daher mußten die gemessenen Reichweiten und Längen der Primär-

s5 Unter Reichweite sei im folgenden die wahre Reichweite verstanden, d.h. die wirkliche Länge der gekrümmten Bahnspur. bahnen durch den Betrag des Expansionsverhältnisses 1,15 dividiert werden.

Um die beobachtete Zahl der Sekundärelektronen mit den Aussagen der Theorie vergleichen zu können, braucht man statt der Reichweite die Energie der Teilchen. Zur Umrechnung steht eine von $\mathrm{Wils}$ o ${ }^{26}$ empirisch gefundene Beziehung $E=$ const $\cdot \sqrt{R}$ zur Verfügung. Die Konstante hat nach $\mathrm{B}$ o th $\mathrm{e}^{27}$ den wahrscheinlichsten Wert const $=23,6\left[\mathrm{keV} \cdot \mathrm{cm}^{-1 / 2}\right]$. Dieses einfache Wurzelgesetz ist allerdings nur bis $E=25 \mathrm{keV}$ hinauf durch Messungen belegt. Bei sehr hohen Energien wird es sicher ungültig und muß durch eine lineare Beziehung zwischen $E$ und $R$ ersetzt werden. Einem solchen Verhalten trägt ein von $\mathrm{Euler}$ und $\mathrm{He}$ isenberg ${ }^{22}$ angegebenes Gesetz Rechnung, welches sich nach entsprechender Umformung und bei geeignet gewählter Konstante schreiben läßt

$E=22,5 \cdot \sqrt{R}+0,5 \cdot R \quad(E$ in $\mathrm{keV}, R$ in $\mathrm{cm})$.

Wenngleich in dem hier interessierenden Bereich von $R$ der Unterschied zum einfachen Wurzelgesetz Wils on s nur geringfügig ist, so wurden doch die Energien nach (3) errechnet.

Anmerkg.b.d.Korr.: Es läßt sich auch aus experimentellen Daten eine Energie-Reichweiten-Funktion gewinnen, und zwar aus dem differentiellen Ionisierungsvermögen und dem mittleren Energieverlust pro Ionenpaar (vergl. W. B o t h e ${ }^{27}$ ). Diese Funktion ist bei Berücksichtigung der Unsicherheiten des experimentellen Materials mit (3) vereinbar.

Abb.11 bringt die Zahl der Sekundärelektronen, die eine Energie $E$ bzw. $E+d E$ besitzen. Sämtliche Häufigkeiten sind in absolutem Maß eingezeichnet, d.h. sie bedeuten die Zahl der Teilchen in einem Energieintervall von $1000 \mathrm{eV}$, bezogen auf $1 \mathrm{~cm}$ Primärbahn. Sehr merkwürdig ist das Ergebnis, daß die Energiespektren der Sekundärelektronen in dem hier untersuchten Bereich für Einzel- und für Schauerstrahlen nicht die gleichen sind. Wohl sind Einzel- und Schauerstrahlen verschiedener Natur - erstere setzen sich bekanntlich in Meereshöhe zu $70 \%$ aus Mesonen zusammen, während nach $\mathrm{Auger} \mathrm{r}^{2,3}$ die Teilchen im ausgedehnten Schauer zu mindestens $90 \%$ Elektronen sind -, doch widerspricht ein unterschiedliches Energiespektrum der Sekun-

26 C. T. R. Wilso n, Proc. Roy. Soc. [London] Ser. A 104, 1 [1923].

${ }_{27}$ W. B othe, Handbuch d. Physik (Ge i g e r Schee l) Bd. 22/2, 2. Aufl. [1933]. 
därelektronen den Erwartungen. Die Stoßtheorie $^{28,29}$ fordert, daß das differentielle Spektrum mit der zweiten Potenz der Energie abfallen soll:

$$
Z(E) d E=\text { const } \cdot E^{-2} d E .
$$

Der Exponent soll unabhängig von der Masse und der Energie (also auch vom Energiespektrum) der stoßenden Teilchen sein. Für Einzelstrahlen ist nach Abb.11 das Gesetz (4) erfüllt. Im Fall der Schauerstrahlen sind jedoch die Meßpunkte nicht mit einem $1 / E^{2}$-Gesetz vereinbar.

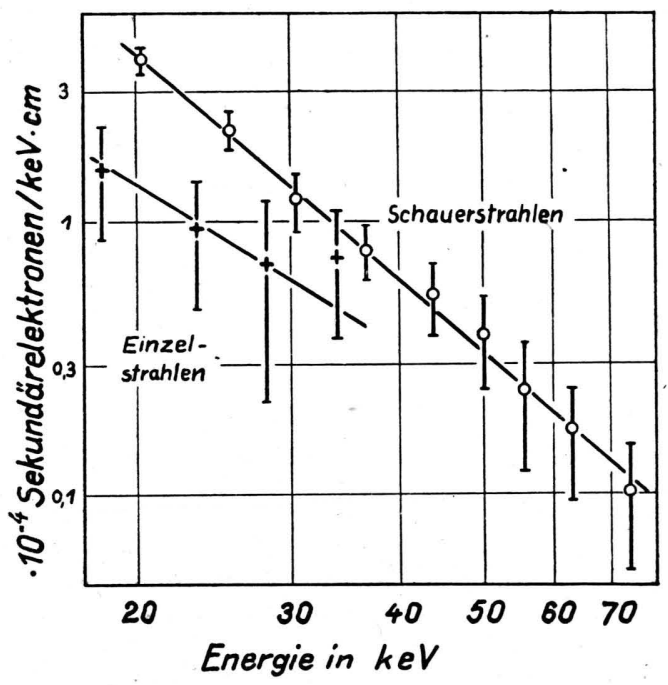

Abb. 11. Häufigkeit der Sekundärelektronen als Funktion ihrer Energie (differentielles Energiespektrum).

Will man sie durch ein Potenzgesetz erklären, so muß der Exponent 2,8 $\pm 0,3$ betragen.

Um der gemessenen steilen Energieverteilung ganz sicher zu sein, wurden nachstehende Proben angestellt: 1. wurde die gesamte, sehr mühselige Ausmessung der Sekundärbahnen wiederholt mit dem gleichen Ergebnis. 2. wurde zur Abschätzung des Einflusses, den systematische Fehler in den Reichweiten ausüben, die größte in Tab. 3 enthaltene Reichweite $(16 \mathrm{~cm})$ willkürlich mit doppelter Größe angesetzt, die kleinste im gleichen Verhältnis verkürzt und die übrigen entsprechend geändert. Der Exponent müßte selbst dann 2,3 lauten. Um 3. den Einfluß des nicht ganz sicheren Energie-Reichweiten-Gesetzes beurteilen

28 Chr. Mølle r, Ann. Physik 14, 531 [1932].

${ }_{29}$ H. B e the, Handbuch d. Physik (G e i g e r S c he e l) Bd. 24/1, 2. Aufl. [1933]. zu können, wurde die Wils o n sche Wurzelformel schon bei $15 \mathrm{keV}$ linear in Richtung der Tangente extrapoliert. Auch dann fiele die Energieverteilung noch mit dèr Potenz 2,4 ab. Weiterhin wurde an folgenden Volumeneffekt gedacht: In den Randgebieten der Kammer kann es vorkommen, daß Sekundärteilchen höherer Energie den Kammerraum verlassen, ihre Reichweite also nicht meßbar wird. Aus diesem Grunde wäre es denkbar, daß die energiereichen Sekundärelektronen z. Tl. verlorengegangen sind, da ihnen ein im Verhältnis zu kleines Kammervolumen zur Verfügung stand. Die genaue Durchrechnung ergibt aber, daß bei der vorliegenden Kammergröße dieser Volumeneffekt den Exponenten des Energiespektrums nur um maximal 0,15 erhöhen könnte. Er war zudem nicht in voller Größe wirksam, denn es wurden auch die Teilchen mitgezählt, deren Bahnende nicht feststellbar war (2 Teilchen mit $R>18 \mathrm{~cm}$ ).

Wie weit stimmt nun unsere Messung mit den Ergebnissen vorhandener Arbeiten überein? Leider gibt es keine entsprechende Untersuchung mit Schauerstrahlen oder ähnlich energiereichen Elektronen als stoßenden Teilchen. Es existieren lediglich Messungen mit $\beta$-Strahlen von Energiebeträgen zwischen 0,5 und $2,6 \mathrm{MeV}$, die jedoch in der Verteilung der Sekundärenergien ganz deutliche Abweichungen von der Theorie zeigen. So beobachten $\mathrm{Shearin}$ und $\mathrm{Pardue} \mathrm{e}^{30}$ an Sekundärelektronen von 20 bis $40 \mathrm{keV}$ ein Energiespektrum, das sich zwischen dem hier gemessenen und dem theoretischen bewegt. W i l liams und Terroux finden von 7,5 bis $40 \mathrm{keV}$ eine Verteilung, welche sich ausgezeichnet mit unserer Messung deckt. In der Absolutzahl der Sekundärteilchen besteht mit beiden Messungen größenordnungsmäßige Übereinstimmung.

Die mit der Theorie übereinstimmende Sekundärelektronenverteilung der Einzelstrahlen ist ebenfalls mit anderen Messungen gut verträglich. N ed d e r m e y er und A n d e r s o n ${ }^{32}$ haben Sekundärteilchen von Einzelstrahlen untersucht, die in $\mathrm{Pb}$ - und Pt-Schichten ausgelöst wurden. Nach dieser Messung bestätigt sich das $1 / E^{2}$-Ge-

${ }^{30}$ P. E. Shearin u. T. E. Pardue,- Physic. Rev. 59, 933 [1941].

31 E. J. Willi a ms u. F. R. Terroux, Proc. Roy. Soc. [London] Ser. A 126, 289 [1930].

${ }_{32}$ S. H. Neddermeyer u. C. D. Anderson, Rev. mod. Physics 11, 203 [1939]. 
setz bis $\mathrm{zu}$ mindestens $150 \mathrm{MeV}$ hinauf. Innerhalb der Genauigkeitsgrenzen dieser und unserer Messungen lassen sich die absoluten Teilchenzahlen pro cm Primärbahn aneinander anschließen, obwohl die Sekundärenergien um drei Zehnerpotenzen auseinander liegen.

Vorliegende Arbeit wurde im Physikalischen Institut der Technischen Hochschule Berlin ausgeführt.
Meines hochverehrten, inzwischen verstorbenen Lehrers, Hrn. Prof. Dr. H. Ge i g e r, gedenke ich in herzlicher Dankbarkeit. Er gab nicht nur die Anregung zu dieser Arbeit, sondern war auch an ihrem Fortgang sehr stark interessiert. Für zahlreiche helfende Ratschläge bin ich Hrn. Dozent Dr. O. Haxel zu Dank verpflichtet. Durch wertvolle Diskussionen mit Hrn. Dozent Dr. F. B o p p wurde die Arbeit weiter gefördert. Der Deutschen Forschungs$\mathrm{g}$ e me in s ch aft danke ich für ein Stipendium.

\title{
Können Protonen als primäre Komponente der kosmischen Strahlung angesehen werden? ${ }^{1}$
}

\author{
Von Karl-Heinz Höcker \\ Aus dem Kaiser-Wilhelm-Institut für Physik, Hechingen \\ (Z. Naturforschg. 2a, 69-72 [1947]; eingegangen am 7. Januar 1947)
}

\begin{abstract}
Es wird versucht, die bisherigen Erfahrungen über die Höhenstrahlung in der Atmosphäre vom Standpunkt einér primären Protonenkomponente zu verstehen. Dabei erweist sich das neutrale Meson als von entscheidender Bedeutung.
\end{abstract}

So eit jetzt mehr als zehn Jahren ist auf Grund von sich allmählich häufenden experimentellen Befunden die These aufgestellt worden, daß Elektronen nicht die primäre Komponente der kosmischen Strahlung sein können, wie man zunächst annahm. An deren Stelle können nach unserem heutigen Wissen lediglich Protonen gesetzt werden. Neutronen und Mesonen dürften ihres radioaktiven Zerfalls wegen dafür nicht in Frage kommen.

Die primären Protonen müssen in der oberen Atmosphäre unter Erzeugung von Mesonen und vielleicht einzelnen Elektronen absorbiert werden, da man Protonen mit beträchtlicher Energie in mäßigen Höhen bereits nicht mehr beobachtet. Man muß sich also vorstellen, daß energiereiche Protonen $\left(E>10^{9} \mathrm{eV}\right)$ durch Stoß- oder StreuProzesse an den Nukleonen (= Protonen + Neutronen) in der Stratosphäre Sekundärteilchen erzeugen. Diese müssen überwiegend Mesonen sein. Lichtquanten und Elektronen können es deshalb nicht (ausschließlich) sein, weil solche Teilchen,

1 Auszug. Eine ausführliche Darstellung erfolgt demnächst an anderer Stelle.

2 M. Schein, W. P. Jesse u. E. O. Woll a n, Physic. Rev. 59, 615 [1941].

3 E. O. Wollan, M. Schein u. W. P. Jesse, Physic. Rev. 59, 930 [1941]. wenn sie die Höhenstrahlung in der Atmosphäre zum Gefolge haben sollen, so energiereich sein müssen, daß sie sogleich Kaskaden bilden. Diese sind aber nicht gefunden ${ }^{2,3,4,5}$. Also müssen $\mathrm{zu}^{-}$ nächst Mesonen entstehen. Die weiche Komponente dürfte dann durch den Zerfall der Mesonen oder aus Anstoßelektronen entstehen. Damit das Maximum der Ionisation ${ }^{6}$ jedoch in nicht zu große Tiefen gerückt wird, darf die Energie der Mesonen nicht groß sein; vielmehr müssen viele Mesonen mit geringer Energie erzeugt werden. Diese muß so gering sein, daß die aus ihnen entstehenden Elektronen keine größeren Kaskaden mehr auslösen können. Denn das Maximum an Teilchen selbst bei kleinen Kaskaden $\left(E_{K} \approx 10^{9} \mathrm{eV}\right)$ tritt erst nach Durchsetzung einer Luftschicht äquivalent $\mathrm{zu}$ einem Druck von etwa $11 \mathrm{~cm} \mathrm{Hg}$ auf, während das Ionisationsmaximum bereits bei $8 \mathrm{~cm} \mathrm{Hg}$ liegt. Mit einer mittleren Mesonenenergie um $9 \cdot 10^{8} \mathrm{eV}$ (Elektronenenergie um $4,5 \cdot 10^{8} \mathrm{eV}$ ) kommt das Ionisationsmaximum in der Atmosphäre etwa an die richtige Stelle. Die Höhe des

6 G. P f ot z e r, Z. Physik 102, 23 [1936].

4 M. Schein u. M. I o na, Physic. Rev. 63, 60 [1943].

5 M. Schein u. J. T a b in, Physic. Rev. 63, 223 [1943]. 